\title{
An efficient Secure Electronic Mail System based on Elliptic Curve Certificateless Signcryption
}

\author{
Roayat Ismail Abdelfattah \\ Electronics and Communication \\ Dept, Faculty of Engineering, \\ Tanta University \\ Tanta, Egypt
}

\author{
Lamiaa Abdelsalam Telb \\ Electronics and Communication \\ Dept, Faculty of Engineering \\ Tanta University \\ Kafrelsheikh, Egypt
}

\author{
Mahmoud Ahmed Attia \\ Electronics and Communication \\ Dept, Faculty of Engineering \\ Tanta University \\ Tanta, Egypt
}

\begin{abstract}
Electronic Mail or E-mail is an important development in the communication world. Therefore, the email security and efficiency has become a critical issue. Most of the existing email systems use either S/MIME (Secure/Multipurpose Internet Mail Extensions) or PGP (Pretty Good Privacy) which depend on Public Key Infrastructure (PKI) or Identity-Based Cryptography (IBC) and use inefficient signature-thenencryption techniques. Each one of these techniques has its own drawbacks. Recently, Certificateless Cryptography (CLC) and Elliptic Curve (EC) based signcrption which combines both signature and encryption in logically one step are developed to overcome these drawbacks with efficient methods. In this paper, a CLC-EC- signcryption based secure E-mail system is proposed. To make the system more efficient, the encryption key is hidden in the transmitted ciphertext itself. The system is highly secure as it uses multi-factor authentication technique includes IP address, password and fingerprint for registration and login. It provides all the security services: confidentiality, integrity, authentication, non-repudiation and forward secrecy with high efficiency compared with other recently existing schemes. Also, it is optionally for the user to send his email in clear form or signcrypted. Finally, it is practically implemented by C\# programming language and it can work on the real network system without changing in the existing network architecture.
\end{abstract}

\section{General Terms}

Security

\section{Keywords}

Certificateless Cryptography; signcryption; Elliptic Curve Cryptography; multi-factor authentication.

\section{INTRODUCTION}

The importance of E-mail is summarized in that the exchange of messages and sensitive documents using computer technology and the Internet easily, instead of paper mail and fax. E-mail started in 1965 at the Massachusetts Institute of Technology (MIT) and it was called MailBox to exchange messages among computers. In 1971, an email system appeared by Ray Tomilson in the department of defense (DoD) and succeeded in sending an email to himself [1]. The existing email system uses the Internet as a transmission medium with standard protocols such as Simple Mail Transfer Protocol (SMTP), the Internet Mail Access Protocol (IMAP) and the Post Office Protocol (POP). But these e-mails can be accessed by attackers, so it has to be protected such that no one can access these messages except the desired people. Any protocol for securing email has to achieve four basic and necessary security requirements, namely, authentication, confidentiality, integrity and nonrepudiation.
The existing e-mail security systems as S/MIME and PGP which based on PKI or IBC are inefficient and suffer from some limitations. For example, PKI suffers [2] from key management problems, complex, expensive certificate management and problems in scalability. To overcome the key management drawback, some email protocols using IBC have been introduced [3-5] .In IBC, the user's email address is used as his public key while a trusted third party named Private Key Generator (PKG) generates private key for each user then transmitted it to users on a secure channel. So IBC has a drawback which is the key escrow problem as the PKG know users' private keys and hence it is able to decrypt any message which violates the nonrepudiation service. In this paper, an email system using a CL-EC signcryption will be introduced to overcome drawbacks of both PKI and IBC based mailing systems and achieves all the required security services [6]. The encryption key is hidden in the transmitted cipher text itself by using an ideas originated from steganography [7-10]. The security of proposed scheme is enhanced using a multi-factor authentication technique for registration and login to the system is used. . The rest of this paper is organized as follows: section 2 presents preliminaries to the CLC, ECC and signcryption, section 3 introduces the proposed system, section 4 introduces simulation results, section 5 gives security analysis, section 6 gives comparison analysis. Finally, section 7 gives conclusions.

\section{PRELIMINARIES}

\subsection{Certificateless Public Key Cryptography (CL-PKC)}

In 2003, CL-PKC has been introduces by Al-Riyami and Paterson [11] to address the drawback of IBC as it has a different scenario. A trusted third party, named the Key Generation Center (KGC) generates the partial private key for each user who then chooses a secret value and combines it with the partial private key to obtain his private key. So the user's private key is unknown to the KGC. By Comparing CL-PKC to IB-PKC, the trust level in the third party is reduced. The KGC has no chance to replace a user's public key due to the used blinding method by the user. CL-PKC [12-15] has more advantages than PKI and IBC so it is an ideal alternative to them.

\subsection{Elliptic Curve Cryptography (ECC)}

PKC system depends on using two separate keys, one public and the other is private for encryption and decryption respectively. The larger the key size the more secure the system is. The most common mathematical hard problems used in PKC are integer factorization and discrete logarithm. These two hard problems are used in RSA and DSA algorithms respectively. In 1985, Miller [16] and Koblitz [17] introduced a new public key cryptography called elliptic curve (EC) which improves the efficiency of various techniques. Actually, cryptographers have 
found that they can achieve computational efficiency in performance and higher security with very low key-size compared to other algorithms. ECC [18-20] uses smaller parameters compared with other competitive algorithms as RSA [21], with the same levels of security. This achieves fewer computations, processing power and storage space. An EC is defined as the set of points given as

$$
\mathrm{y}^{2}=\mathrm{x}^{3}+\mathrm{ax}+\mathrm{b}
$$

Where $4 a^{3}+27 b^{2} \neq 0(\operatorname{modp})$. This is named EC Weierstrass normal form as shown in Fig. 1.

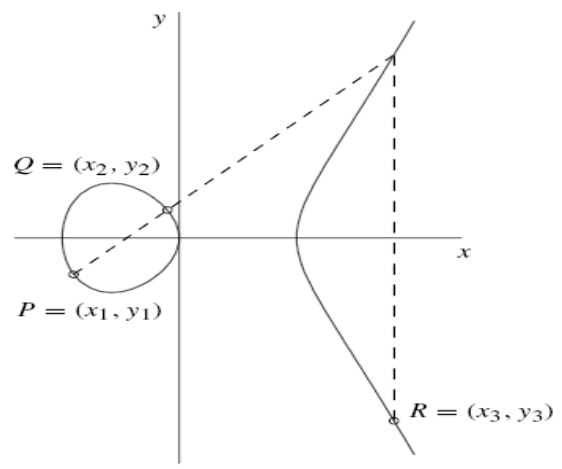

Figure 1: Addition: $R=P+Q$

\section{- EC point addition:}

If $\mathrm{P}\left(\mathrm{x}_{1}, \mathrm{y}_{1}\right)$ and $\mathrm{Q}\left(\mathrm{x}_{2}, \mathrm{y}_{2}\right)$ are two points on an $\mathrm{EC}$ and $\mathrm{P}\left(\mathrm{x}_{1}, \mathrm{y}_{1}\right)$ $\neq \mathrm{Q}\left(\mathrm{x}_{2}, \mathrm{y}_{2}\right)$, by drawing line between $\mathrm{P}$ and $\mathrm{Q}$ it will intersect the EC at a third point $\mathrm{R}(\mathrm{x} 3, \mathrm{y} 3)$. The addition of $\mathrm{P}$ and $\mathrm{Q}$ is reflection of this point about $\mathrm{X}$-axis. It is calculated using following equations:

$\mathrm{x}_{3}=\lambda^{2}-\mathrm{x}_{1}-\mathrm{x}_{2}$

$\mathrm{y}_{3}=\lambda\left(\mathrm{x}_{1}-\mathrm{x}_{3}\right)-\mathrm{y}_{1}$

And $\lambda=\frac{\mathrm{y}_{2}-\mathrm{y}_{1}}{\mathrm{x}_{2}-\mathrm{x}_{1}}$

if $P \neq Q$

EC Discrete Logarithm Problem EC-DLP:

For a point $\mathrm{P} \square \square \mathrm{E}(\mathrm{Fq})$ of order $\mathrm{n}$, where $\mathrm{E}$ is an $\mathrm{EC}$ defined over a finite field $\mathrm{Fq}$, and another point $\mathrm{Q} \square \square \mathrm{E}(\mathrm{Fq})$, the $\mathrm{EC}$ DLP is to find the integer $\mathrm{k} \square \square[0, \mathrm{n}-1]$ such that $\mathrm{Q}=\mathrm{k}$ P. The integer $\mathrm{k}$ is named the $\mathrm{DL}$ of $\mathrm{Q}$ to the base $\mathrm{P}$, denoted $\mathrm{k}=\log \mathrm{P}$ Q.

$\mathrm{Q}=\mathrm{kP}$ is called scalar point multiplication, which consume the most time in ECC computations. The private key of each user is chosen randomly while the public key is the multiplication of the private key with a generator point $\mathrm{G}$ on the EC.

\subsection{Signcryption}

Zheng in 1997 [22] introduces a cryptographic scheme, called signcryption, that merges the digital signature and the encryption into one logical step, so requires a smaller computational cost compared to encryption then signature systems. This signcryption achieved 50\% fewer computational cost and $85 \%$ fewer communication overhead. Since then, many signcryption schemes have been introduced [23-29].

\subsection{Multi-factor authentication (MFA) Technique}

MFA achieves a second level of security during logging in. It prevents unauthorized users from logging into the accounts and hence protects identities, data, money...etc. When logging in, a user is required to enter a password and also authenticate him using a second factor. There are many types of authentication factors as passwords, smart cards, biometrics (face-eye-handfingerprint...ect) and a user's location information (example: GPS, IP address). The proposed scheme uses IP address; password and fingerprint as MFA.

\section{THE PREPOSED SYSTEM}

The proposed scheme includes three parties: Key Generator Center (KGC) calculates the partial private key for all users when they register in the system, the sender who signcrypt the desired message and the receiver who unsigncrypt the received message. It consists of two phases:

- Offline phase: the users do it without having a message to send.

- Online phase: the users do it when they have a message to send.

The block diagram of sending and receiving a message with the proposed scheme is shown in Figure 2 and Figure. 3.

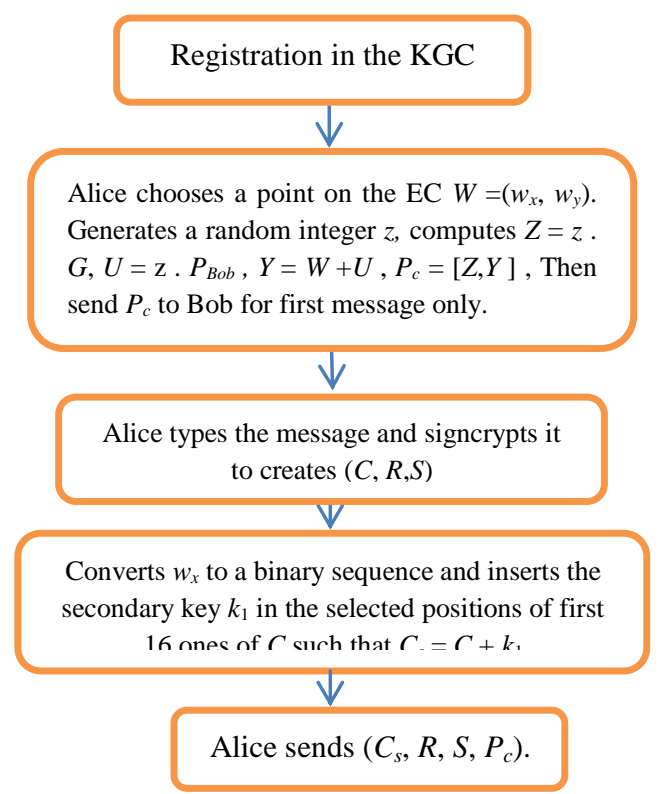

Figure 2: Sending secure email

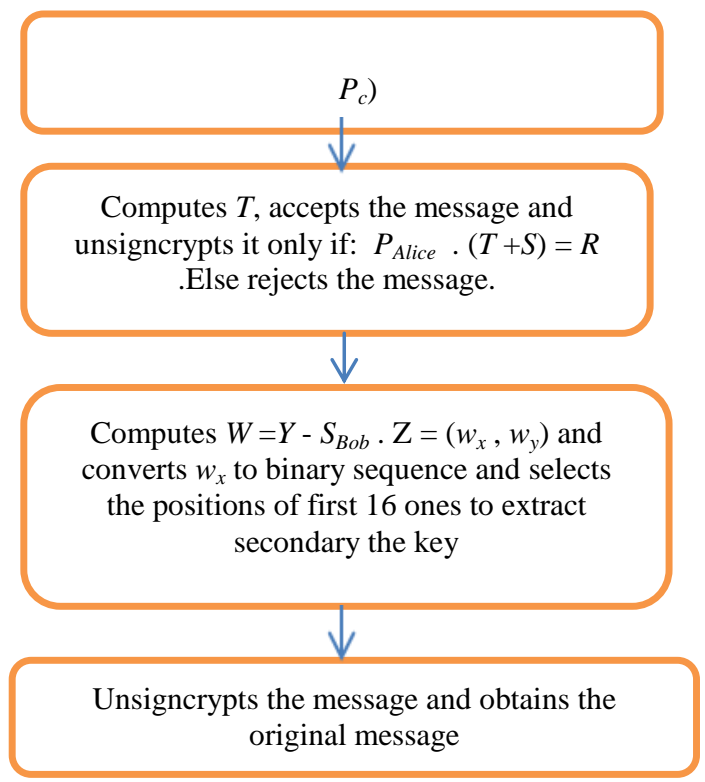

Figure 3: Receiving secure email 


\subsection{Offline phase}

This phase makes the proposed scheme more efficient. It consists of three sub phases: registration, key generation, and transmission of master secret key

\subsubsection{Registration phase}

All users have to register to the KGC sever by providing multifactor authentication (user ID, password and finger print) to enable the user to login every time.

\section{Key generation phase}

It includes two major steps: selection of domain parameters and key generation.

\subsubsection{Selection of domain parameters}

The parameters are:

$\mathrm{p}$

$\mathrm{a}, \mathrm{b}$

F

$\infty$

G

n

$\mathrm{H}$

$\mathrm{E}_{\mathrm{k}}(.) / \mathrm{D}_{\mathrm{k}}($.$) \quad The encryption/decryption scheme which is AES-$ 128 bit.

\subsubsection{Key Generation}

The proposed scheme uses the same steps of Al-Riyami and Paterson Scheme [11]; which consists of five steps: setup, secret value set, extraction of partial private key, private and public keys set.

Setup: This step is performed by the KGC which do the following:

1. Select a generator $\mathrm{G} \in \mathrm{F}$.

2. Select a master secret-key $\mathrm{s}<\mathrm{p}$, and compute public key of KGC

$$
\mathrm{P}_{0}=\mathrm{s} . \mathrm{G}
$$

Secret value set: This step is performed by the user who do the following:

1. User $m$ that has identifier $\mathrm{ID}_{\mathrm{m}}$ selects $\mathrm{x}_{\mathrm{m}}<\mathrm{p}$.

2. Computes his/her public key:

$$
\mathrm{X}_{\mathrm{m}}=\mathrm{x}_{\mathrm{m}} \cdot \mathrm{G}
$$

$$
\text { and sends } X_{m} \text { to the KGC. }
$$

a. Extraction of partial private key: The KGC constructs the partial private key for user $\mathrm{m}$ whose identifier $\mathrm{ID}_{\mathrm{m}}$ as following:

1. Calculate:

$$
\mathrm{Q}_{\mathrm{m}}=\mathrm{H}\left(\mathrm{ID}_{\mathrm{m}} \| \mathrm{X}_{\mathrm{m}}\right)
$$

2. Calculate the partial private key:

$\mathrm{D}_{\mathrm{m}}=\mathrm{s} \cdot \mathrm{Q}_{\mathrm{m}}$ b. Private key set: In this step the user $m$ does the following:

Compute the private key $S_{m}$ from the partial private key $D_{m}$ as following:

$\mathrm{S}_{\mathrm{m}}=\mathrm{x}_{\mathrm{m}} \cdot \mathrm{D}_{\mathrm{m}}=\mathrm{x}_{\mathrm{m}} \mathrm{s} \cdot \mathrm{Q}_{\mathrm{m}}$

c. Public key set: the user $m$ constructs his public key as: $P_{m}=S_{m} \cdot G$

Finally the user $\mathrm{m}$ has one key pair $\left(\mathrm{S}_{\mathrm{m}}, \mathrm{P}_{\mathrm{m}}\right)$

Assume Alice is the receiver who with a private key, $\mathrm{S}_{\text {Alice }}=$ $\mathrm{x}_{\text {Alice }} \cdot \mathrm{D}_{\text {Alice }}$, and a public key $\mathrm{P}_{\text {Alice }}=\mathrm{S}_{\text {Alice }} \cdot \mathrm{G}$, while the receiver Bob with a private key, $\mathrm{S}_{\mathrm{Bob}}=\mathrm{x}_{\mathrm{Bob}} \cdot \mathrm{D}_{\mathrm{Bob}}$, and a public key, $\mathrm{P}_{\mathrm{Bob}}=\mathrm{S}_{\mathrm{Bob}}$.G. All users' public keys are available on the KGC's secure web site.

In the proposed scheme two secret keys will be used for encryption: the primary key and the secondary key. The primary key is constant for all the messages and is used to determine the positions of the ciphertext where the secondary key is hidden, while the secondary key is a varying with each message and it is hidden in the ciphertext. The message will be encrypted with the secondary key.

\subsubsection{Transmission of primary secret}

EC encryption is used once for transmission of primary secret key as follows:

At the sender (Alice)

1. Select a random point on the elliptic curve $\mathrm{W}=\left(\mathrm{w}_{\mathrm{x}}\right.$, $\mathrm{w}_{\mathrm{y}}$ ) where $\mathrm{w}_{\mathrm{x}}$ is used as the primary secret key.

2. Convert $\mathrm{w}_{\mathrm{x}}$ to binary sequence then determine the positions of first 16 ones. this places will be the positions in the ciphertext where the secondary key bytes will be inserted. In every position of ones in $w_{x}$ insert one byte of the secondary key in the ciphertext To send the primary key $\mathrm{w}_{\mathrm{x}}$ securely to the receiver, EC encryption will be used as following:

Generate a random number $\mathrm{z}$ and compute:

$$
\begin{aligned}
& Z=z \cdot G \\
& U=z \cdot P_{\text {Bob }} \\
& Y=W+U
\end{aligned}
$$

$P_{c}=[Z, Y]$ is the encrypted primary secret key which Alice sends to Bob only one time before the existence of any message.

At the receiver: Upon receiving $\mathrm{P}_{\mathrm{c}}=[\mathrm{Z}, \mathrm{Y}]$, Bob compute $\mathrm{W}=\mathrm{Y}-\left(\mathrm{S}_{\mathrm{Bob}} \cdot \mathrm{Z}\right)=\left(\mathrm{w}_{\mathrm{x}}, \mathrm{w}_{\mathrm{y}}\right)$

Then convert $\mathrm{w}_{\mathrm{x}}$ to binary sequence to determine the ones positions.

\subsection{Online phase}

This phase consists of two sub phases: signcryption and unsigncryption.

\subsubsection{Signcryption}

When Alice has a message $\mathrm{M}$ to send in an E-mail to Bob, she does the following:

1. Choose a random integer $\mathrm{x}<\mathrm{p}$.

2. Compute Hash value of $x$ with SHA-256 and divide it into two blocks $\mathrm{k}_{1}$ and $\mathrm{k}_{2}$ each of 128 bit length. $\mathrm{k}_{1}$ will be used as the secondary secret key for message encryption with AES-128: 


$$
\mathrm{C}=\mathrm{E}_{\mathrm{k}_{1}}(\mathrm{M})
$$

3. $\mathrm{k}_{1}$ will be hidden in the ciphertext by using the primary $\mathrm{w}_{\mathrm{x}}$ as follow : $\mathrm{k}_{1}=128$ bit $=16$ byte, these bytes will be inserted in the ciphertext $\mathrm{C}$ in the first 16 positions of ones in $\mathrm{w}_{\mathrm{x}}$. If number of ones in $\mathrm{w}_{\mathrm{x}}$ less than 16 in this case insert the reminder of 16 bytes of $\mathrm{k}_{1}$ in the end of $\mathrm{C}$. Now the transmitted ciphertext will be $\mathrm{C}_{\mathrm{s}}=\mathrm{C}+\mathrm{k}_{1}$.

4. Select random number $\mathrm{v}<\mathrm{p}$.

5. Compute:

$$
\begin{gathered}
\mathrm{R}=\mathrm{v} \cdot \mathrm{G}=\left(\mathrm{R}_{\mathrm{x}}, \mathrm{R}_{\mathrm{y}}\right) \\
\mathrm{T}=\operatorname{Hash}\left(\mathrm{C}_{\mathrm{s}} / / \mathrm{R}_{\mathrm{x}}\right)
\end{gathered}
$$

symbol

means

$\mathrm{S}=\left(\mathrm{v} / \mathrm{S}_{\text {Alice }}-\mathrm{T}\right) \bmod \mathrm{p}$

the concatenation.

Then Alice sends $\left(\mathrm{C}_{\mathrm{s}}, \mathrm{R}, \mathrm{S}\right)$ to Bob.

\subsubsection{UnSigncryption phase}

Upon receiving $\left(C_{s}, R, S\right)$, Alice does the following:

1. Compute:

$\mathrm{T}=\operatorname{Hash}\left(\mathrm{C}_{\mathrm{s}} / / \mathrm{R}_{\mathrm{x}}\right)$

2. Accept $\left(\mathrm{C}_{\mathrm{s}}\right)$ only if: $\mathrm{P}_{\text {Alice }} \cdot(\mathrm{T}+\mathrm{S})=\mathrm{R}$ else reject the message.

3. $\operatorname{Extract}\left(\mathrm{k}_{1}\right)$ from $\left(\mathrm{C}_{\mathrm{s}}\right)$ where $\mathrm{C}=\mathrm{C}_{\mathrm{s}}-\mathrm{k}_{1}$

4. Decrypt $(\mathrm{C})$ to obtain the message as:

$\mathrm{M}=\mathrm{E}_{\mathrm{k}_{1}}(\mathrm{C})$

\section{SIMULATION RESULTS}

Proposed scheme is implemented in software using C\# programming language with windows 64-bit operating system, processor Intel (R) Core(TM) i5-3340M CPU @2.70 GHz and memory (RAM) $4.00 \mathrm{~GB}$. The results are displayed in the figures from Figure 4 to Figure 15.

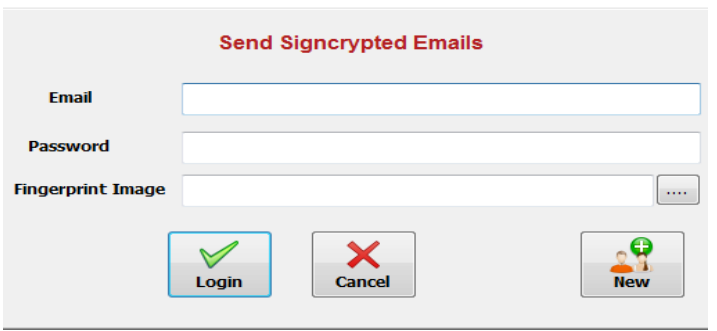

Figure 4: Snapshot of send receive program.

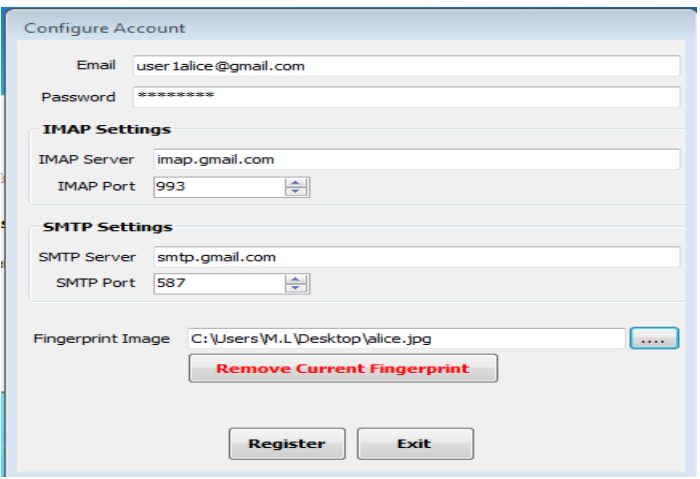

Figure 5: The first join of the user to the system press New button $\stackrel{2}{-}$, enters his data then press Register to join to KGC.

\section{Send Signcrypted Emails}

Email

user1alice@gmail.com|

Password

\section{***********}

Fingerprint Image C:|Users|M.LLDesktop|alice.jpg
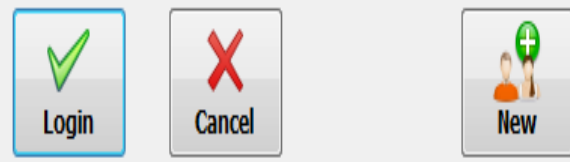

Figure 6: The user that already registers on the KGC enters his Email ID, password, and his finger print image, then press log in button.

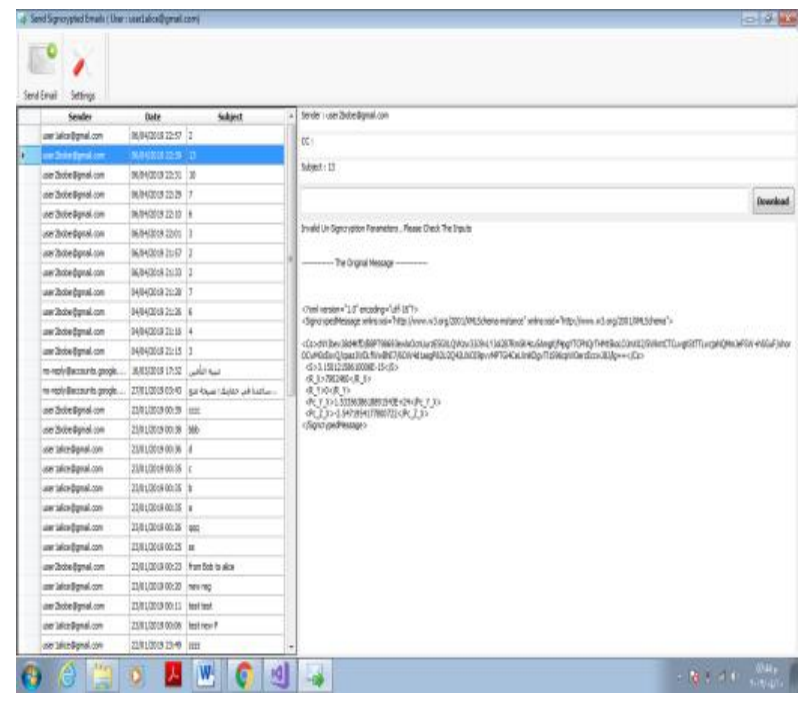

Figure 7: Send- receive program contents of two menus.

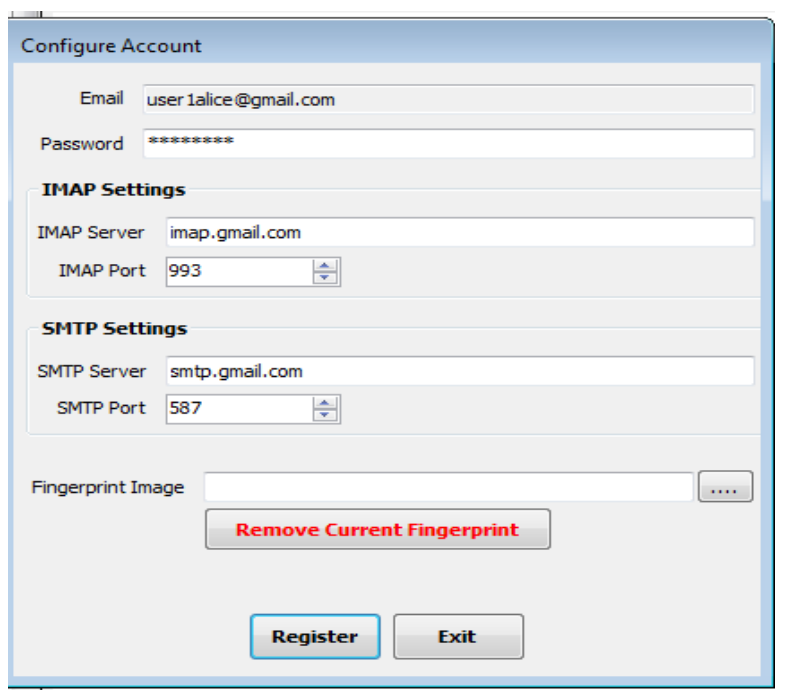

Figure 8: Settings menu 


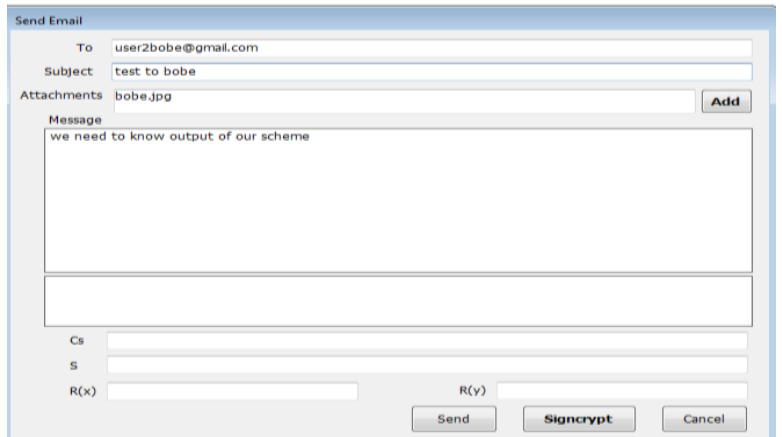

Figure 9: Send email that used for sending the desired email without signcryption.

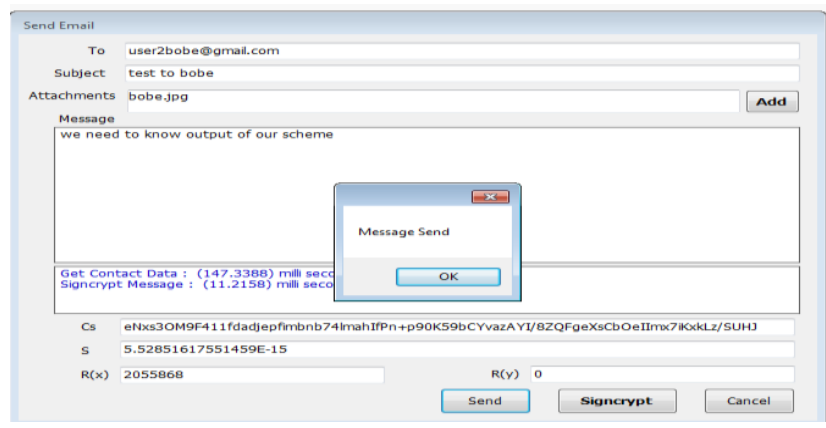

Figure 10: After signcryption of the message, press on "Send" button to send the signcrypted message to the receiver that is already register on the web site of key generation center.

Note that in the snapshot of signcryption time is about 11 mill seconds, while the same signcrypted message in [30] takes 197 mills second which proves that proposed scheme is very efficient.

If the signature is correctly verified, the receiver will decrypt the ciphertext to recover the original message. While if the signature isnt verified, a warning message will appear and so the ciphertext will not be decrypted.

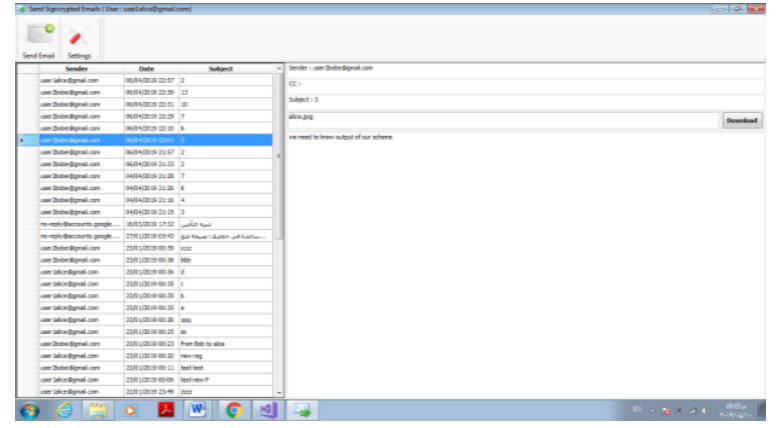

Figure 11: Snapshot of verified signature

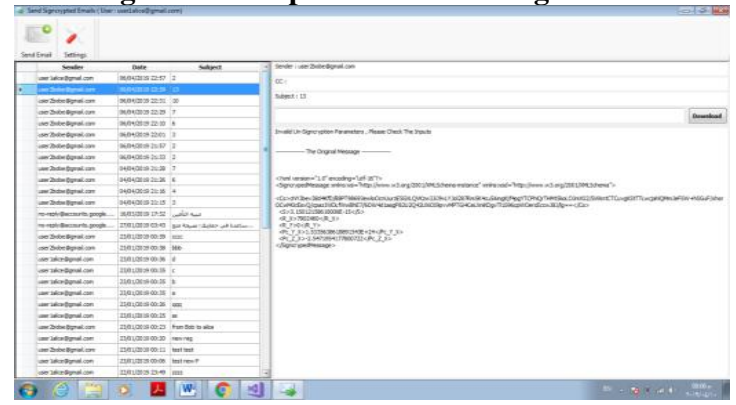

Figure 12: Snapshot of unverified signature with changing only one byte of $S$

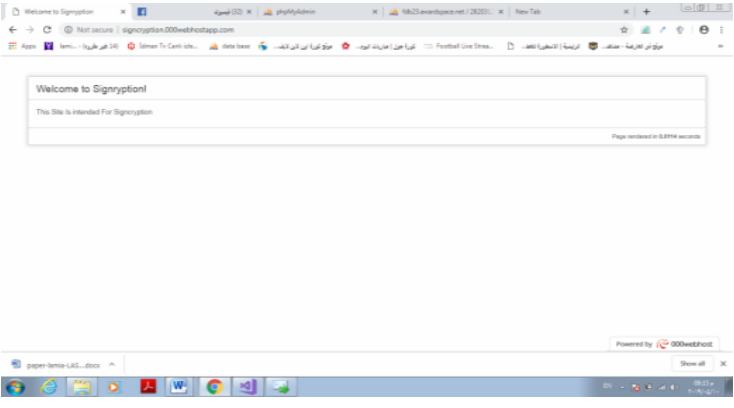

Figure 13: Snapshot of web site of KGC

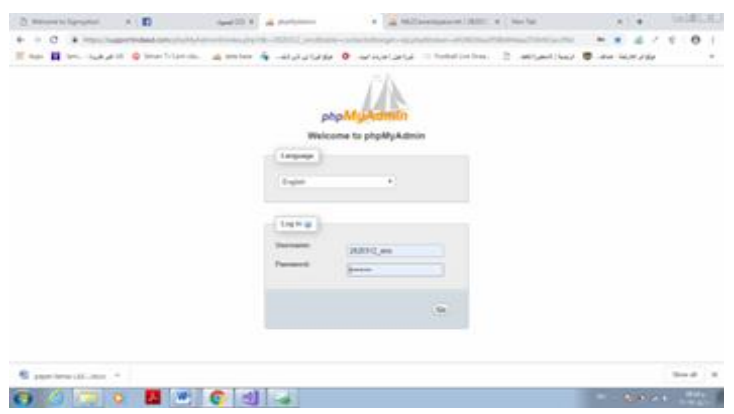

Figure 14: Snapshot of database of KGC

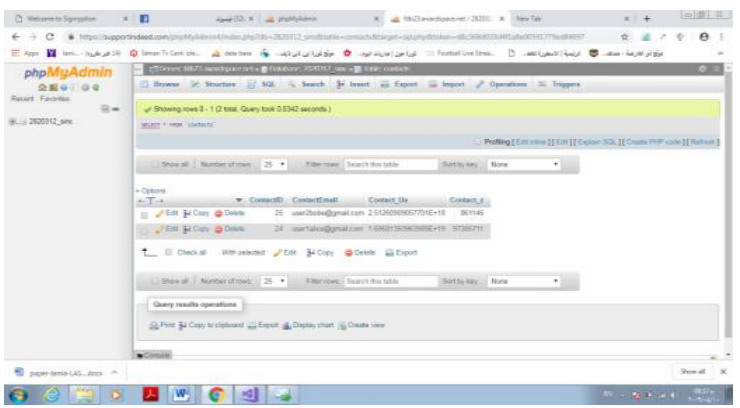

Figure 15: Snapshot of data base of key generation center

\section{SECURITY ANALYSIS}

The proposed scheme is an EC-DLP based scheme. For an entity $\mathrm{m}$ whose public key is

$P_{m}=S_{m}$. G, where $S_{m}$ is his/her private key and $G$ is the generator of the EC, then if both $\mathrm{S}_{\mathrm{m}}$ and $\mathrm{G}$ are given, the public key $\mathrm{P}_{\mathrm{m}}$. can be easily computed. But given the public key $\mathrm{P}_{\mathrm{m}}$ and $\mathrm{G}$, the computation of the private key $\mathrm{S}_{\mathrm{m}}$ is computationally hard. This is known as the EC-DLP. The proposed scheme achieves all security services as follows:

\subsection{Confidentiality}

In the proposed scheme, there are two secret keys: the primary key $\mathrm{w}_{\mathrm{x}}$ and the secondary key $\mathrm{k}_{1}$. The secondary key $\mathrm{k}_{1}$ is inserted in the ciphertext in positions depending on the ones in the binary sequence of the primary key. So proposed scheme security depends totally on the primary key $\mathrm{w}_{\mathrm{x}}$ which is protected by the EC-DLP.

The attacker has access to only $\mathrm{C}_{\mathrm{s}}, \mathrm{R}, \mathrm{S}$ and $\mathrm{P}_{\mathrm{C}}$. To obtain $\mathrm{w}_{\mathrm{x}}$ he has to know $\mathrm{z}$ or $\mathrm{S}_{\mathrm{Bob}}$ which are protect by EC-DLP according to Eqns. 11,12,13, and 14 .

\subsection{Message integrity and non-repudiation}

From the digital signature equation $S=\left(v / S_{\text {Alice }}-T\right) \bmod p$ the sender Alice signs using his private key $\mathrm{S}_{\text {Alice }}$ and he/she cannot disown sending the email message. The receiver verifies if 
$\mathrm{P}_{\text {Alice }} \cdot(\mathrm{T}+\mathrm{S})=\mathrm{R}$. If it is verified, then $\left(\mathrm{C}_{\mathrm{s}}, \mathrm{R}, \mathrm{S}\right)$ is actually sent by the sender Alice. Else the sender did not send it.

\subsection{Public verification}

Proposed scheme achieves public verification for the signature. This means that anyone can be a judge and can verify that the sender actually sent the message. The receiver sends (Cs, R, S) to the judge who can do the following:

1)Calculate $T=\operatorname{Hash}\left(C_{\mathrm{s}} / / R_{\mathrm{x}}\right)$.

2) If $P_{\text {Alice }} \cdot(T+S)=R$, then the judge will be sure that sender actually sent $\left(C_{s}, R, S\right)$ to the receiver, other else the sender did not send it.

Here, all variables that are used in the judge verification are public.

\subsection{Forward Secrecy}

Forward secrecy means even though the attacker obtained the sender's private key, he cannot recover plaintext M. In the propose scheme, if the attacker tries to derive the plaintext $\mathrm{M}$ from $\left(C_{s}, R, S\right)$, he must obtain the secondary key secret $k_{1}$ which is hidden in ciphertext according to the ones positions of the binary sequence of the primary key $\mathrm{w}_{\mathrm{x}}$. To obtain $\mathrm{w}_{\mathrm{x}}$ he needs to know $\mathrm{W}=\mathrm{Y}-\mathrm{U}=\mathrm{Y}-\left(\mathrm{z} \cdot \mathrm{P}_{\mathrm{Bob}}\right)=\mathrm{Y}-\left(\mathrm{S}_{\mathrm{Bob}} \cdot \mathrm{Z}\right)=\left(\mathrm{w}_{\mathrm{x}}\right.$, $\mathrm{w}_{\mathrm{y}}$ ) where $\mathrm{W}$ is independent on sender's private key and protected by EC encryption. Therefore, the proposed scheme achieves forward secrecy.

\subsection{Firewall effect}

The proposed scheme provides a firewall effect which means that the signature is verified before decryption. Upon receiving the ciphertext $\left(\mathrm{C}_{\mathrm{s}}, \mathrm{R}, \mathrm{S}\right)$, the receiver firstly verifies if $\left(\mathrm{P}_{\text {Alice }}\right.$. $(\mathrm{T}$ $+S)=R$ ). Then decrypt $C_{s}$ to obtain the plaintext, else he rejects it without decryption.

\section{COMPARISON}

Here, a comparison of proposed scheme with similar existing schemes will be given. The schemes in $[13,31]$ use the pairing [32] and encryption-then -signature which is inefficient. The scheme in [33] uses certificate cryptography that has some problems and doesn't achieve public verification property. The scheme in [30] also uses pairing so it is inefficient. The scheme in [37] also uses pairing and depend on integer factorization problem which is inefficient .The comparison is shown in Table 1 and Fig. 16. Mul. means No. of point multiplication.

Table 1: Comparison between of the proposed system with other existing schemes

\begin{tabular}{|c|c|c|c|c|c|}
\hline Scheme & Mul. & Firewall & Pairing & $\begin{array}{c}\text { Public- } \\
\text { key } \\
\text { system }\end{array}$ & $\begin{array}{c}\text { Public } \\
\text { verification }\end{array}$ \\
\hline $\begin{array}{c}\text { Scheme1 } \\
\text { in [33] }\end{array}$ & 5 & NO & NO & CA & NO \\
\hline $\begin{array}{c}\text { Scheme2 } \\
\text { in [30] }\end{array}$ & 8 & NO & YES & CLC & NO \\
\hline $\begin{array}{c}\text { Scheme3 } \\
\text { in [34] }\end{array}$ & 9 & NO & NO & CLC & NO \\
\hline $\begin{array}{c}\text { Scheme4 } \\
\text { in [35] }\end{array}$ & 6 & YES & NO & CA & YES \\
\hline $\begin{array}{c}\text { Scheme5 } \\
\text { in [36] }\end{array}$ & 8 & NO & NO & CLC & NO \\
\hline $\begin{array}{c}\text { Proposed } \\
\text { Scheme }\end{array}$ & 3 & YES & NO & CLC & YES \\
\hline
\end{tabular}

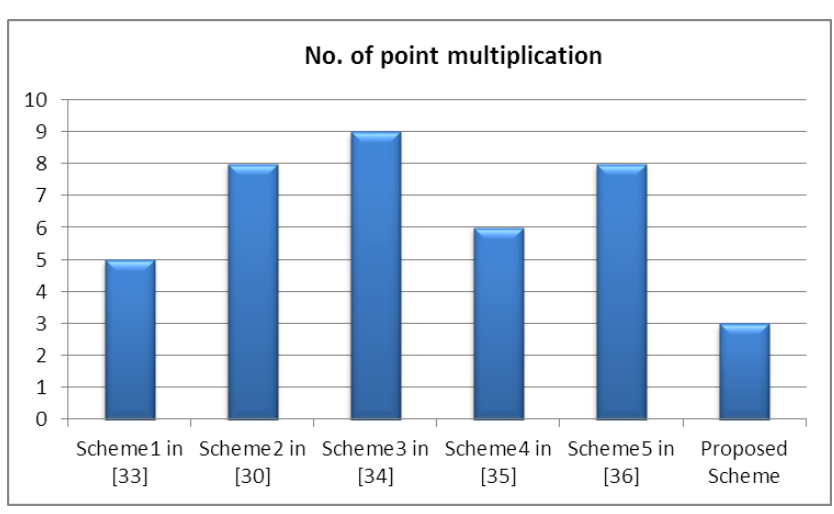

Figure 16: proposed scheme compared with other similar existing schemes.

\section{CONCLUSION}

An efficient secure email scheme based on CL-EC signcryption is introduced in this paper. It achieves all the required security services in an efficient method. The scheme can be applied on the real network system without making remarkable modifications to the currently existing email system. The proposed signcryption is optional and it is implemented using C\# programming language. Security analysis of the proposed scheme has been provided. According to the results, proposed scheme is more efficient than the existing email schemes due to its least number of EC point multiplications without bilinear pairings operation which is time consuming.

\section{REFERENCES}

[1]. J. B. Postel, "Simple mail transfer protocol", 1982. http://www.rfc-editor.org/info/rfc821.

[2]. Buchmann, Johannes A., Karatsiolis, Evangelos, Wiesmaier, Alexander, "Introduction to Public Key Infrastructures " () Springer-Verlag Berlin Heidelberg 2013.

[3]. https://en.wikipedia.org/wiki/ID-based_cryptography

[4]. A. Shamir, "Identity-based cryptosystems and signature schemes", in Advances in Cryptology-CRYPTO'84, 1984, pp. 47-53.

[5]. Ays s e G"ul Karatop and Erkay Savas, "An Identity-Based Key Infrastructure Suitable for Messaging Applications" Faculty of Engineering and Natural Sciences Sabanci University Istanbul, Turkey.

[6]. S. William, "Cryptography and Network Security", vol. 139, no. 3. Boston, USA. Prentice Hall, 2011.

[7]. Khalil Challita and Hikmat Farhat," Combining Steganography and Cryptography: New Directions " (IJNCAA) 1(1): 199-208, 2011 (ISSN 2220-9085).

[8]. Venkata Bhanu Chowdary Allada, Mallikarjun Susarla "Developing an Efficient Solution to Information Hiding through Text Steganography Along with Cryptography", IJCST Vol. 8, Issue 1, Jan - March 2017.

[9]. Amal Khalifa "LSBase: A key encapsulation scheme to improve hybrid crypto-systems using DNA steganography" Conference Paper · November 2013.

[10]. Magdy Saeb,"Encryption Key Distribution Applying Steganographic Techniques", (IJCSCS) August 2014. 
[11]. S. Al-Riyami and K. Paterson, "Certificateless public key cryptography", In C. Laih, editor, Asiacrypt 2003, Lecture Notes in Computer Science, pages 452-473, 2003.

[12]. A. R. Sattam and P. Kenneth,"Certifcateless public key cryptography a full version," in Asiacrypt'03,LNCS 2894, Springer, pp. 452- 473, 2003.

[13]. Suresh Kumar Balakrishnan and V. P. Jagathy Raj "Practical Implementation of a Secure Email System Using Certifcateless Cryptography and Domain Name System", International Journal of Network Security, Vol.18, No.1, PP.99-107, Jan. 2016.

[14].Sattam S. Al-Riyami and Kenneth G. Paterson "Certificateless Public Key Cryptography", Advances in Cryptology - ASIACRYPT 2003 pp 452-473.

[15].Alexander W. Dent,"A Brief Introduction to Certificateless Encryption Schemes and their Infrastructures", EuroPKI 2009: Public Key Infrastructures, Services and Applications pp 1-16.

[16].Miller M.,"Uses of elliptic curves in cryptography". Advances in Cryptography Crypto '85.1986; 417-426.

[17].Koblitiz N., "Elliptic curve cryptosystems". Mathematics of computation. Vol. 48; No. 177; 1987; 203-208.

[18].D. Hankerson, A. J. Menezes, and S. Vanstone, "Guide to Elliptic Curve Cryptography", Springer-Verlag New York Inc, 2006

[19].Jeffrey L. Vagle, "A Gentle Introduction to Elliptic Curve Cryptography", November 21, 2000.

[20].Joseph H. Silverman,"An Introduction to the Theory of Elliptic Curves", 2006.

[21]. https://simple.wikipedia.org/wiki/RSA_algorithm.

[22].Y. Zheng, "Digital signcryption or how to achieve cost (signature \& encryption) $\ll \operatorname{cost}$ (signature) + cost (encryption)," in Seventeenth Annual International Cryptology Conference, 1997, pp. 165-179.

[23].JM L., Mao W., " Two birds one stone: signcryption using RSA". In Topics in Cryptology (CT-RSA'03), Joye M (ed), LNCS 2612. Springer-Verlag: San Francisco, CA, USA, 2003; 211-225.

[24].Malone-Lee J, "Identity based signcryption". Available from: http://eprint.iacr.org/2002/098.pdf [Accessed on 30 May 2011].

[25]. Libert B., Quisquator JJ., "A new identity based signcryption scheme from pairings". In Proceedings of IEEE Information Theory Workshop (ITW'03). Elsevier: Paris, France, 2003; 155-158.

[26]. Chow SSM, Yiu SM, Hui LCK, Chow KP," Efficient forward and provably secure ID based signcryption scheme with public verifiability and public ciphertext authenticity". In Proceedings of Information Security and Cryptology (ICISC'03), Lim JI, Lee DH (eds), LNCS 2971. Springer-Verlag: Seoul, Korea, 2004; 352-369. International Journal of Computer Applications (0975 8887) Volume 165 - No.2, May 201743.

[27].Boyen X., "Multipurpose identity based signcryption: a Swiss army knife for identity based cryptography". In Advance in Cryptology (CRYPTO'03), Boneh D (ed), LNCS 2729. Springer-Verlag: Santa Barbara, California, USA, 2003; 383-399.

[28].Chen L., Malone-Lee J. ,"Improved identity-based signcryption". In Public Key Cryptography (PKC'05), Vaudenay S (ed), LNCS 3386. Springer-Verlag: Les Diablerets, Switzerland, 2005; 362-379.

[29].PSLM B., Libert B., McCullagh N., JJ. Quisquater JJ., "Efficient and provably-secure identity based signatures and signcryption from bilinear maps". In Advance in Cryptology (ASIACRYPT'05), Roy BK (ed), LNCS 3788. Springer-Verlag: Chennai, India, 2005; 515-532.

[30]. Abdul Wahid, Masahiro Mambo," Implementation of Certificateless Signcryption based on Elliptic Curve Using Java script" (IJCANDI) Vol 1, No 3, August 2016, pp. 90100.

[31]. Mohammed Hassouna, Nashwa Abbas Farah, Bazara Barry ,and Eihab Bashier Mohammed Bashier, and Eihab Bashier Mohammed Bashier, "An End-to-End Secure Mail System Based on Certificateless Cryptography in the Standard Security Model",( IJCSI) Vol. 10, Issue 2, No 3, March 2013.

[32].Zhengjun Cao, Lihua Liu,"On the Disadvantages of Pairing-based Cryptography".

[33].A K Mohapatra,PhD, Jyoti Kushwaha,and Tanya Popli "Enhancing Email Security by Signcryption based on Elliptic Curve" , International Journal of Computer Applications (0975 - 8887) Volume 71- No.17, June 2013.

[34].Hui-fang YU, Bo YANG,"Low-computation certificateless hybrid signcryption scheme", Yu and Yang / Front Inform Technol Electron Eng 2017 18(7):928-940.

[35].Malik ZiaID, Rashid Ali ,"Cryptanalysis and improvement of an elliptic curve based signcryption scheme for firewalls", https://doi.org/10.1371/journal.pone.0208857 December 13, 2018

[36].Bo Zhang , Zhongtian Jia, and Chuan Zhao,"An Efficient Certificateless Generalized Signcryption Scheme", https://doi.org/10.1155/2018/3578942, Volume 2018, Article ID 3578942, 11 pages.

[37].Balasubramanian V and Mala T" Improved Certificateless Signcryption for IoT Smart Devices ", Appl. Math. Inf. Sci. 13, No. 1, 31-38 (2019) . 